
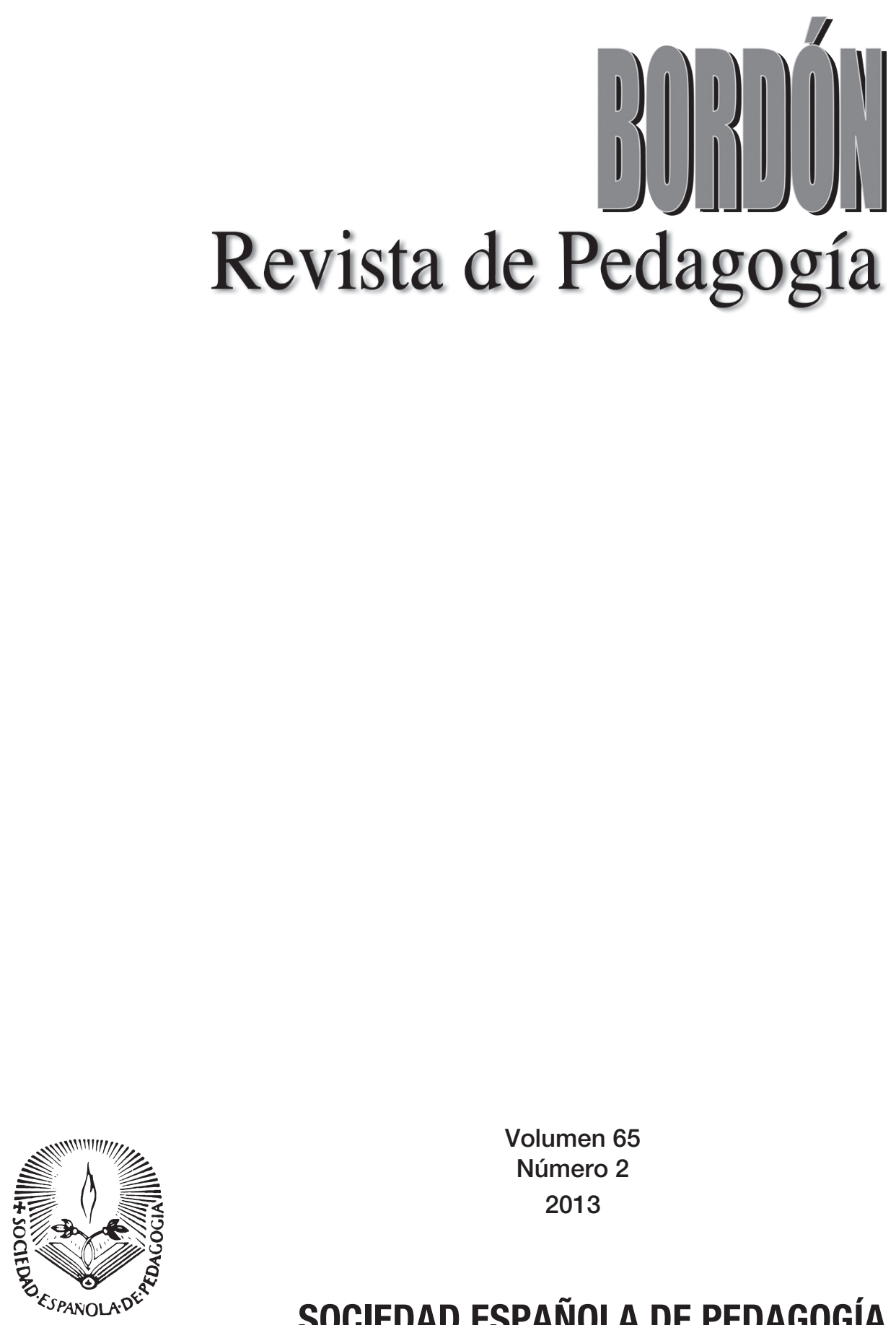

Volumen 65

Número 2

2013

SOCIEDAD ESPAÑOLA DE PEDAGOGÍA 



\title{
AnImACIÓn HOSPITALARIA CON PACIEnTES ADULTOS EN EL HOSPITAL LA FE DE UALENCIA: UN ESTUDIO DE MECESIDADES
}

\section{Socio-Cultural Animation with Adult Patients at La Fe Hospital [Ualencia, Spain]: a Meeds Analysis}

\author{
Ma TERESA BERMÚDEZ REY \\ Universidad de Oviedo \\ $M^{a}$ EUGENIA MARTÍN PALACIO \\ Universidad Complutense de Madrid \\ SILVIA CASTELLANOS CANO \\ Universidad de Oviedo
}

INTRODUCCIÓN. En este artículo se muestran los resultados de una investigación realizada sobre animación hospitalaria con pacientes adultos en el Hospital de La Fe (Valencia), que tenía como objetivo mostrar la necesidad de ocupar el tiempo libre de los mismos. MÉTODO. Se administró un cuestionario a una muestra de 96 pacientes. El cuestionario hacía referencia al uso del tiempo libre del paciente en el hospital, a la propuesta de actividades de animación y a las posibles consecuencias derivadas de las mismas. RESULTADOS. Se obtuvieron correlaciones positivas entre la edad del paciente y el desinterés a la hora de realizar actividades y negativas entre la edad y la preferencia por la oferta de actividades. Se obtuvieron correlaciones positivas entre el tiempo de hospitalización y el uso del tiempo libre (pasear, charlar), entre el tiempo de hospitalización y la preferencia por la oferta de actividades de difusión cultural y acompañamientos profesionales y entre este tiempo y las consecuencias percibidas por el paciente. DISCUSIÓN. Se contempla el interés de los pacientes por realizar actividades en proporción inversa a su edad.

Palabras clave: Animación sociocultural, Ocio, Pacientes, Adultos.

\section{Introducción}

En Perspectivas actuales de la animación (Ventosa, 2006) se recoge el tema de la animación hospitalaria, destacándose allí que esta se diseña para pacientes hospitalizados que disponen de mucho tiempo libre (tiempo del que dispone el paciente después de realizadas las visitas médicas, el descanso o la alimentación), sin embargo, la animación hospitalaria ha sido estudiada desde una perspectiva pediátrica $y$, frecuentemente, no profesionalizadora, pero está escasamente tratada desde el punto de vista de la hospitalización adulta.

Algunos estudios que inciden en esta cuestión de forma directa o indirecta son: Sarrate (1998), en tanto que ya destacaba a la animación hospitalaria como espacio emergente en la animación 
sociocultural, visión posteriormente avalada por las numerosas actividades realizadas por el voluntariado en los hospitales, principalmente con pacientes pediátricos; Costa (2000), quien publica estudios sobre actividades lúdicas en el hospital, suponiendo su libro El juego y el juguete en la hospitalización infantil una importante referencia en este ámbito; Bados (2004), que aboga por una política de animación sociocultural sistematizada que haga olvidar el voluntarismo y la precariedad del trabajo de estos profesionales en los hospitales; Bermúdez y Torío (2007) y Manzanera, Ullán y Gómez (2008), quienes abordan, entre otros, el tema de la animación con pacientes pediátricos y la necesidad de contratar a profesionales que realicen labores de animación hospitalaria; Ullán y cols. (2010), quienes constatan la baja representación de los adolescentes en los informes sobre hospitalización y servicios de salud, y ponen de manifiesto cómo los mismos sujetos proponen mejorar su estancia en los hospitales a través de los servicios de ocio, aportando un punto de vista muy interesante sobre la necesidad de extender dicha animación no solo al paciente pediátrico, sino también a una población difícil de clasificar dentro de la animación hospitalaria realizada hasta la fecha; sobre el empleo del tiempo libre del paciente adulto en el hospital, se están llevado a cabo trabajos financiados por la Universidad de Oviedo en el Hospital Universitario Central de Asturias (Fernández, Torío y Bermúdez, 2010), que han confirmado la necesidad de avalar la realización de actividades de animación con estos enfermos y, finalmente, cabe señalar la escasa representación de los estudios relacionados con la hospitalización y la animación en la tercera edad (Bermúdez, 2009), si bien, existe una amplia tradición de animación con pacientes de estas edades en otros contextos, como residencias de tercera edad, centros de día, etc., fácilmente extrapolable al ámbito hospitalario.

\section{Objetivos}

De lo anteriormente expuesto cabe concluir que los estudios sobre proyectos de animación sociocultural con pacientes adultos en el hospital no son muy abundantes. Tomando en consideración este vacío, el presente artículo muestra los resultados de una investigación realizada con enfermos adultos en el Hospital de La Fe de Valencia, durante el mes de mayo de 2011.

Su objetivo principal es mostrar la necesidad de ocupar el tiempo libre de los mismos, partiendo de la hipótesis de que esta ocupación genera efectos beneficiosos para los pacientes. Y son objetivos adjuntos establecer diferencias significativas de esta necesidad en función de las variables edad y permanencia en el centro hospitalario.

\section{Método}

Para la realización de este trabajo se ha empleado una metodología de carácter empírico con recogida de información individualizada, siguiendo un protocolo preestablecido de preguntas cerradas.

\section{Muestra}

Se ha tenido acceso a 96 pacientes adultos con distintos tiempos de hospitalización que estaban ingresados en el Hospital de la Fe de Valencia, en el periodo comprendido entre el 2 y el 10 de mayo de 2011 y en distintas unidades: Lesionados Medulares, Medicina Interna, Rehabilitación, Neurología y Neurocirugía, Hematología y Traumatología (véase tabla 1).

Los enfermos se seleccionaron teniendo en cuenta su condición de pacientes adultos no ambulatorios y que estuvieran en buenas condiciones físicas y mentales para responder a las preguntas formuladas.

\section{Instrumento}

Se empleó un cuestionario de 12 ítems que contemplaba básicamente cuatro apartados: a) datos sociodemográficos; b) ocupación del 
tiempo libre del paciente en el hospital (actividades que realiza en él y espacios en donde las lleva a cabo); c) propuestas de actividades desde la animación hospitalaria para realizar en el tiempo libre; d) consecuencias que podrían colegirse del desarrollo de las mismas.

El cuestionario se realizó teniendo en cuenta por tanto el sexo y la edad del paciente hospitalizado; el nivel de estudios realizados (básico, medio o superior); el tiempo de hospitalización (considerándose hospitalizaciones cortas las de una semana o menos, largas las comprendidas entre una semana y un mes, y muy largas las que excedían ese periodo); las actividades que realizaba a lo largo del día una vez concluidas las visitas médicas (ver la televisión, escuchar música, hablar por teléfono, leer, etc.); los espacios en las que realizaba estas actividades; la percepción del paciente sobre la conveniencia de ofertar actividades de animación por parte del hospital; las posibles consecuencias derivadas de su aplicación y, por último, el interés que podría demostrar el paciente por realizar alguna de las actividades propuestas (lectura guiada, creación de grupos de humor, talleres de distinto tipo, etc.).

Con el fin de facilitar la contestación del cuestionario, el paciente debía marcar las opciones que consideraba convenientes en cada uno de lo ítems.

\section{Procedimiento de recogida de la información}

Durante los primeros meses de 2011, se contactó con la Gerencia del Hospital La Fe de Valencia y se tramitaron los permisos pertinentes para acceder a las habitaciones de los pacientes adultos.

En coordinación con la Gerencia del Hospital y el Servicio de Enfermería, se tuvo acceso a las habitaciones de pacientes adultos ingresados en las unidades de Lesionados Medulares, Medicina Interna, Rehabilitación, Neurología, Hematología y Traumatología.
Esta labor no estuvo exenta de dificultades, solventadas mayormente por el buen hacer de los profesionales de la enfermería del hospital, que facilitaron de forma considerable el acceso a las habitaciones, la presentación de la tarea a cada paciente $y$, en suma, el proceso de recogida de datos.

De esta forma, la investigadora firmante en primer lugar del trabajo se reunió con la coordinadora y con los enfermeros encargados de cada planta, presentando la tarea a realizar. Posteriormente, el profesional encargado de cada servicio la acompañó a cada una de las habitaciones previamente seleccionadas, realizando ante el paciente una breve síntesis del objeto de estudio. Esta explicación se amplió por parte de la investigadora, quien a continuación depositó los cuestionarios en las habitaciones, volviendo a recogerlos al cabo de media hora.

En todo momento se tuvo especial cuidado en no molestar a los pacientes y en no transgredir la rutina hospitalaria de cada planta.

\section{Análisis de datos}

Para el estudio de las relaciones se utilizó el paquete estadístico SPSS (Statistical Package for the Social Sciences), versión 18.0. Se llevaron a cabo correlaciones entre las respuestas dadas por los pacientes y las variables (edad, tiempo de hospitalización), utilizando el coeficiente de correlación de Pearson para describir la relación directa o inversa entre las mismas.

\section{Resultados}

Se han establecido correlaciones entre la edad y la realización de actividades habituales en el hospital (ver la televisión, escuchar música, navegar por Internet, hablar por teléfono, hacer pasatiempos, leer, pasear, charlar, o no realizar actividad alguna), obteniéndose una correlación positiva entre los pacientes que no 
realizan actividades y la edad, de forma que a mayor edad más desinterés se observa por parte de los enfermos para realizar actividades en el tiempo libre en el hospital.

Sin embargo, se han obtenido correlaciones negativas entre ver la televisión y edad, de forma que a menor edad los pacientes se distraen más viendo la televisión y a la inversa; entre navegar por Internet y la edad, de forma que a menor edad más se interesa el paciente por llevar a cabo estas actividades en el hospital y a la inversa, y entre hablar por teléfono y edad, ya que a menor edad más se habla por teléfono y a la inversa (véase tabla 2).

Igualmente, se han establecido correlaciones entre edad y lugar de desarrollo de las actividades por parte del paciente en el hospital (habitación, sala compartida, pasillos y otros), obteniéndose una correlación negativa entre la edad y el desarrollo de actividades en lugares poco transitados por los pacientes, de tal forma que a menor edad más se transita por lugares poco frecuentados del hospital (véase tabla 3).

Se han establecido correlaciones entre la edad y la conveniencia de ofertar actividades en los centros sanitarios (acompañamiento de profesionales, grupos de charla guiada, lecturas y comentarios, proyección de películas, conferencias, juegos de mesa, risoterapia, actividades con radio o televisión, actividades con ordenador e Internet, celebración de fiestas, actuaciones de personajes famosos, visitas de pacientes adultos a pediátricos, realización de periódicos y/o revistas hospitalarias, así como talleres de distinto tipo), obteniéndose una correlación negativa entre la oferta de películas en sala por parte del hospital y la edad, de tal forma que a menor edad más preferencia existe por la oferta de películas en sala y a la inversa; entre la oferta de juegos de mesa y la edad, de tal forma que a menor edad más se valora la oferta de juegos de mesa por parte del hospital y a la inversa; entre la preferencia por realizar actividades de radio o televisión en el hospital y la edad, de tal forma que a menor edad más interés existe por ellas y a la inversa; la preferencia por el uso de Internet en el hospital y la edad, de forma que a menos edad, más interés se manifiesta por las mismas y a la inversa; la preferencia de actuaciones por parte de personajes relevantes en el hospital y la edad, de forma que a menos edad más interés existe por ellas y a la inversa, y la preferencia en la oferta de distintos talleres (barro, dibujo...) en el hospital y la edad, de forma que a menos edad, más interés existe por ellos y a la inversa (véase tabla 4).

Se han observado correlaciones entre el tiempo de hospitalización y las actividades que realiza el paciente en el tiempo libre durante su estancia en el hospital (ve la televisión, escucha música, navega por Internet, habla por teléfono, realiza pasatiempos, lee, pasea, charla o no realiza actividad alguna), así existe una correlación positiva entre el tiempo de hospitalización y pasear en el hospital, de forma que a más edad, más se pasea; y entre el tiempo de hospitalización y charlar en el hospital, de forma que a más edad, más comunicación verbal se mantiene con otras personas (véase tabla 5).

Se constatan correlaciones entre el tiempo de hospitalización y los lugares que transita el paciente durante su estancia en el hospital (habitación, sala compartida, pasillos y otros), observándose que existe una correlación positiva entre el tiempo de hospitalización y el uso de la sala compartida, de forma que a más tiempo de hospitalización, más se utiliza ésta (véase tabla 6).

Asimismo, se han establecido correlaciones entre el tiempo de hospitalización y el tipo de actividad deseada (acompañamientos profesionales, grupos de charla guiada, lectura y comentarios de prensa, proyección de películas, conferencias, juegos de mesa, risoterapia, actividades con radio y/o televisión, actividades con ordenador/ Internet, celebración de fiestas, actuaciones con personajes famosos, visita de pacientes adultos a pediátricos, realización de revistas y/o periódicos, talleres de dibujo, barro...), encontrándose que 
existe una correlación positiva entre el tiempo de hospitalización y la preferencia por el acompañamiento de profesionales, de manera que a mayor tiempo de hospitalización más se prefiere que el hospital oferte acompañamientos de este tipo; entre el tiempo de hospitalización y la preferencia por actividades de difusión cultural como conferencias en el hospital, de forma que a más tiempo de hospitalización, más se demandan, y, finalmente, se ponen de manifiesto correlaciones negativas entre el tiempo de hospitalización y la celebración de fechas señaladas, de manera que a más tiempo de hospitalización, menor preferencia existe hacia la oferta de actividades de este tipo (véase tabla 7).

Se observan correlaciones entre el tiempo de hospitalización y las posibles consecuencias derivadas de la oferta de actividades de animación en el hospital, encontrándose una correlación positiva entre el tiempo de hospitalización y la comunicación que establece el paciente, de forma que a más tiempo de hospitalización, más se cree que las actividades de animación hospitalaria favorecen la comunicación entre paciente/ profesional y/o paciente/paciente en el hospital; entre el tiempo de hospitalización y la adaptación al hospital, de forma que a más tiempo de hospitalización, más se cree que la oferta de actividades de animación mejora la adaptación del paciente al hospital y, finalmente, entre tiempo de hospitalización y atención integral al paciente, de forma que a más tiempo de hospitalización, más se considera que las actividades de animación pueden propiciar la atención integral al paciente hospitalizado (véase tabla 8).

\section{Conclusiones}

Con respecto a las correlaciones establecidas a cerca de la edad y realización de actividades, se obtiene que a mayor edad más desinterés existe por realizar algún tipo de actividad que comporte distracción en el hospital y, puesto que la oferta de las mismas por parte de los hospitales es mínima o inexistente, tampoco al paciente se le pone en la tesitura de elegir sobre una base real; el caso de pacientes pediátricos (e incluso el de los adolescentes) transita por vías completamente opuestas, dado que se ofertan y cuentan con una gran aceptación entre los enfermos (Bermúdez y Torío, 2007).

Los pacientes adultos de más edad ven menos tiempo la televisión, ocupándolo por tanto en descansar o pensar, probablemente en términos negativos sobre su enfermedad; por otra parte, los pacientes adultos más jóvenes muestran preferencia por ver la televisión, de lo que cabe inferir que las actividades propuestas sobre los medios audiovisuales pueden ser igualmente aceptadas por estos.

Abundando en la circunstancia del aislamiento del paciente, se constata que a mayor edad menos se habla por teléfono y menos actividades relacionadas con Internet se realizan; en este sentido (y sin olvidar cuestiones tan estudiadas como la referida a la brecha digital entre grupos de edad), hay que mencionar que los ordenadores utilizados por los pacientes son particulares, puesto que el hospital no dispone de salas con este cometido para adultos y no siempre se cuenta con una conexión a Internet (en clara oposición a las actividades realizadas con pacientes pediátricos en las denominadas ciber@ulas hospitalarias); sin embargo, se cree que esta práctica es especialmente recomendable para que los pacientes se distraigan y no pierdan contacto con el exterior, especialmente si se ven sometidos a hospitalizaciones de larga duración.

De ahí que se pueda concluir que el paciente adulto de mayor edad (no así el resto) ingresado en el hospital disminuye sus actividades de distracción al máximo, mostrando escaso interés por realizar otras; todo hace suponer que permanece inactivo transitando por los pasillos y por la habitación y/o charlando, actividades claramente insuficientes para ocupar todo el día, máxime si su estancia en el centro es prolongada. 
También interesa mencionar que uno de los usos habituales del ocio es el relacionado con el afrontamiento del dolor, experiencia subjetiva que si no se trata puede contribuir a que se desarrollen problemáticas asociadas (depresión, alteraciones del sueño, aumento de peso, reducción de la movilidad, disminución de la socialización), sin embargo, cuando el paciente se involucra en una actividad placentera, su dolor no es el foco principal de atención, por ello es importante contemplar una oferta de actividades significativas para el enfermo que distraigan su atención (Martínez y Amayra, 2006).

Entre los aspectos investigados en torno al ocio se encuentran las emociones que produce: humor, sensación de implicación, activación, relax, percepción del tiempo, ideas, creencias, autoconciencia, etc. Vera (2006), haciéndose eco de los estudios de Peterson, Seligman y Vaillant (1988), señala que el optimismo (entendemos también el derivado de la puesta en marcha de distintas actividades) palía el sufrimiento y malestar de aquellos que sufren a causa de la enfermedad.

Por lo que respecta al tiempo de hospitalización, se observa, como cabía esperar, que a mayor tiempo de estancia en el hospital más se valoran las ofertas de actividades de animación por parte del centro, y también se observa que éstas podrían ayudar en la adaptación del paciente al centro sanitario.

A esto habría que añadir la diferente percepción del tiempo por parte de los enfermos, así Bayés (2003) distingue entre tiempos subjetivos y tiempos cronológicos en los pacientes: para los pacientes la vivencia temporal se dilata y la incertidumbre en los tiempos de espera hace que estos se alarguen igualmente. Para este autor, existe mucho sufrimiento en los hospitales, que podría, entendemos, paliarse de forma sencilla a través de programas de animación hospitalaria.

De igual forma, a mayor tiempo de hospitalización más se demanda el acompañamiento por parte de profesionales, y las actividades de difusión cultural en el hospital que perciben los enfermos favorecen la atención integral del paciente en el entorno sanitario, y contribuyen a hacer más fluida la comunicación entre los pacientes y entre los pacientes y los profesionales.

Mencionar, por tanto, que la calidad de vida en el hospital está estrechamente relacionada con el ocio, por lo que la persona necesita tanto de ese tiempo como de su ocupación en actividades que le reporten descanso y distracción, alejándolo, además, de las rutinas dirigidas propias de las instituciones hospitalarias; para Gonzalo y otros (2008) el hecho de participar de las actividades de ocio durante los periodos de hospitalización incrementa el grado de satisfacción y facilita la adopción de actitudes positivas en el paciente, posibilitando que el grado de actividad y también la autoestima se mantenga alta.

No hay que olvidar, por último, dos hechos que consideramos importantes: por un lado la mayoría de los pacientes ingresados en los hospitales son (y serán en un futuro) adultos, puesto que a la par que aumenta la esperanza de vida se incrementa el número de enfermedades crónicas y de hospitalizaciones de larga duración; y por el otro, el hecho recurrente en la puesta en marcha de proyectos de animación sociocultural, a saber: son las personas que más lo necesitan las que muestran mayor desinterés en formar parte de ellos, debido a sus peculiares circunstancias, y precisamente por ello se hace más necesaria la oferta y difusión de un amplio abanico de actividades de animación en los centros sanitarios. 


\section{Anexo}

\section{TABLA 1. Muestra estudiada}

\begin{tabular}{llc}
\hline \multirow{2}{*}{ Sexo } & Hombres & $53,1 \%$ \\
\cline { 2 - 3 } Edad & Mujeres & $46,9 \%$ \\
\cline { 2 - 3 } & Menor 30 años & $9,4 \%$ \\
\cline { 2 - 3 } & 30-50 años & $26,6 \%$ \\
\cline { 2 - 3 } Tiempo hospitalización & Mayor 70 años & $37,6 \%$ \\
\hline Servicios hospital & Corto & $26,0 \%$ \\
\cline { 2 - 3 } & Largo & $56,3 \%$ \\
\cline { 2 - 3 } & Muy largo & $27,1 \%$ \\
\cline { 2 - 3 } & Lesionados medulares & $16,7 \%$ \\
\cline { 2 - 3 } & Medicina Interna & $7,5 \%$ \\
\cline { 2 - 3 } & Rehabilitación & $3,3 \%$ \\
\cline { 2 - 3 } & Neurología y neurocirugía & $16,4 \%$ \\
\cline { 2 - 3 } & Hematología & $21,9 \%$ \\
\cline { 2 - 3 } & Traumatología & \\
\hline
\end{tabular}

TABLA 2. Correlación entre edad del paciente y realización de actividades habituales en el hospital

Actividades

\begin{tabular}{|c|c|c|}
\hline \multirow{3}{*}{ Ninguna } & Correlación de Pearson &, $224(*)$ \\
\hline & Sig. (bilateral) & ,028 \\
\hline & $\mathrm{N}$ & 96 \\
\hline \multirow[t]{3}{*}{ Ve la televisión } & Correlación de Pearson &,$- 306(* *)$ \\
\hline & Sig. (bilateral) & 002 \\
\hline & $\mathrm{N}$ & 96 \\
\hline \multirow[t]{3}{*}{ Escucha música } & Correlación de Pearson &,- 176 \\
\hline & Sig. (bilateral) &, 087 \\
\hline & $\mathrm{N}$ & 96 \\
\hline \multirow[t]{3}{*}{ Internet } & Correlación de Pearson &,$- 266(* *)$ \\
\hline & Sig. (bilateral) &, 009 \\
\hline & $\mathrm{N}$ & 96 \\
\hline \multirow[t]{3}{*}{ Habla por teléfono } & Correlación de Pearson &,$- 209\left(^{*}\right)$ \\
\hline & Sig. (bilateral) & ,041 \\
\hline & $\mathrm{N}$ & 96 \\
\hline
\end{tabular}


TABLA 2. Correlación entre edad del paciente y realización de actividades habituales en el hospital (cont.)

Actividades

Hace pasatiempos

Correlación de Pearson

$-, 083$

Sig. (bilateral)

, 420

$\mathrm{N}$

96

Lee

Correlación de Pearson

$-, 089$

Sig. (bilateral)

, 386

$\mathrm{N}$

96

Pasea

Correlación de Pearson

$-, 079$

Sig. (bilateral)

, 446

Charla

$\mathrm{N}$

Correlación de Pearson

96

Sig. (bilateral)

$-, 094$

$\mathrm{N}$

Correlación de Pearson

,363

Otros

Sig. (bilateral)

96

$\mathrm{N}$

, 145

* La correlación es significante al nivel 0,05 (bilateral).

** La correlación es significativa al nivel 0,01 (bilateral).

TABla 3. Correlaciones entre la edad del paciente y espacios utilizados para realizar actividades en el hospital

Actividades

Habitación

Correlación de Pearson

Sig. (bilateral)

$\mathrm{N}$

Sala compartida

Pasillos

Otros

Correlación de Pearson $\quad-, 191$

Sig. (bilateral) $\quad 062$

$\mathrm{N} \quad 96$

Correlación de Pearson $\quad-, 129$

Sig. (bilateral) , 210

$\mathrm{N} \quad 96$

Correlación de Pearson $\quad-, 246(* *)$

Sig. (bilateral) ,016

$\mathrm{N}$

* La correlación es significante al nivel 0,05 (bilateral).

** La correlación es significativa al nivel 0,01 (bilateral). 
TABla 4. Correlación entre la edad del paciente y la preferencia de supuestas actividades de animación en el hospital

\section{Preferencias}

\begin{tabular}{|c|c|c|}
\hline \multirow{3}{*}{ Acompañamiento por profesionales } & Correlación de Pearson & , 179 \\
\hline & Sig. (bilateral) & 081 \\
\hline & $\mathrm{N}$ & 96 \\
\hline \multirow{3}{*}{ Grupos de charla guiada } & Correlación de Pearson &,- 003 \\
\hline & Sig. (bilateral) & ,976 \\
\hline & $\mathrm{N}$ & 96 \\
\hline \multirow[t]{3}{*}{ Lectura y comentarios } & Correlación de Pearson &,- 005 \\
\hline & Sig. (bilateral) & ,965 \\
\hline & $\mathrm{N}$ & 96 \\
\hline \multirow[t]{3}{*}{ Proyección de películas } & Correlación de Pearson &,$- 213(*)$ \\
\hline & Sig. (bilateral) &, 037 \\
\hline & $\mathrm{N}$ & 96 \\
\hline \multirow[t]{3}{*}{ Conferencias } & Correlación de Pearson &,- 160 \\
\hline & Sig. (bilateral) &, 120 \\
\hline & $\mathrm{N}$ & 96 \\
\hline \multirow[t]{3}{*}{ Juegos de mesa } & Correlación de Pearson &,$- 359(* *)$ \\
\hline & Sig. (bilateral) &, 000 \\
\hline & $\mathrm{N}$ & 96 \\
\hline \multirow[t]{3}{*}{ Risoterapia } & Correlación de Pearson &, 037 \\
\hline & Sig. (bilateral) &, 719 \\
\hline & $\mathrm{N}$ & 96 \\
\hline \multirow[t]{3}{*}{ Actividades con radio o TV } & Correlación de Pearson &,$- 212(*)$ \\
\hline & Sig. (bilateral) &, 038 \\
\hline & $\mathrm{N}$ & 96 \\
\hline \multirow{3}{*}{$\begin{array}{l}\text { Actividades con ordenador } \\
\text { o Internet }\end{array}$} & Correlación de Pearson &,$- 347(* *)$ \\
\hline & Sig. (bilateral) &, 001 \\
\hline & $\mathrm{N}$ & 96 \\
\hline \multirow[t]{3}{*}{ Celebración fiestas } & Correlación de Pearson &,$- 361(* *)$ \\
\hline & Sig. (bilateral) &, 000 \\
\hline & $\mathrm{N}$ & 96 \\
\hline \multirow[t]{3}{*}{ Actuaciones famosos } & Correlación de Pearson &,$- 304(* *)$ \\
\hline & Sig. (bilateral) &, 003 \\
\hline & $\mathrm{N}$ & 96 \\
\hline \multirow{3}{*}{$\begin{array}{l}\text { Visitas de pacientes adultos } \\
\text { a pacientes pediátricos }\end{array}$} & Correlación de Pearson &,- 044 \\
\hline & Sig. (bilateral) &, 667 \\
\hline & $\mathrm{N}$ & 96 \\
\hline
\end{tabular}


TABLA 4. Correlación entre la edad del paciente y la preferencia de supuestas actividades de animación en el hospital (cont.)

Preferencias

Realización de periódicos o revistas

Correlación de Pearson

, 025

Sig. (bilateral) ,809

$\mathrm{N} \quad 96$

Talleres (dibujo, barro...)

Correlación de Pearson $\quad-, 224\left({ }^{*}\right)$

Sig. (bilateral) ,029

N 96

* La correlación es significante al nivel 0,05 (bilateral)

** Significativa nivel 0,01 bilateral.

Tabla 5. Correlación entre el tiempo de hospitalización y actividades habituales del paciente hospitalizado

Actividades

\begin{tabular}{|c|c|c|}
\hline \multirow[t]{3}{*}{ Ninguna } & Correlación de Pearson &,- 010 \\
\hline & Sig. (bilateral) & ,920 \\
\hline & $\mathrm{N}$ & 96 \\
\hline \multirow[t]{3}{*}{ Ve la televisión } & Correlación de Pearson &, 021 \\
\hline & Sig. (bilateral) & ,836 \\
\hline & $\mathrm{N}$ & 96 \\
\hline \multirow[t]{3}{*}{ Escucha música } & Correlación de Pearson &, 027 \\
\hline & Sig. (bilateral) & ,797 \\
\hline & $\mathrm{N}$ & 96 \\
\hline \multirow[t]{3}{*}{ Internet } & Correlación de Pearson &, 145 \\
\hline & Sig. (bilateral) &, 159 \\
\hline & $\mathrm{N}$ & 96 \\
\hline \multirow[t]{3}{*}{ Habla por teléfono } & Correlación de Pearson &, 099 \\
\hline & Sig. (bilateral) & ,338 \\
\hline & $\mathrm{N}$ & 96 \\
\hline \multirow[t]{3}{*}{ Hace pasatiempos } & Correlación de Pearson &, 177 \\
\hline & $\underline{\text { Sig. (bilateral) }}$ &, 084 \\
\hline & $\mathrm{N}$ & 96 \\
\hline \multirow[t]{3}{*}{ Lee } & Correlación de Pearson &, 096 \\
\hline & Sig. (bilateral) & 351 \\
\hline & $\mathrm{N}$ & 96 \\
\hline
\end{tabular}


Tabla 5. Correlación entre el tiempo de hospitalización y actividades habituales del paciente hospitalizado (cont.)

Actividades

\begin{tabular}{|c|c|c|}
\hline \multirow[t]{3}{*}{ Pasea } & Correlación de Pearson &, $272(* *)$ \\
\hline & Sig. (bilateral) &, 007 \\
\hline & $\mathrm{N}$ & 96 \\
\hline \multirow[t]{3}{*}{ Charla } & Correlación de Pearson &, $235(*)$ \\
\hline & Sig. (bilateral) &, 021 \\
\hline & $\mathrm{N}$ & 96 \\
\hline \multirow[t]{3}{*}{ Otros } & Correlación de Pearson &, 192 \\
\hline & Sig. (bilateral) &, 061 \\
\hline & $\mathrm{N}$ & 96 \\
\hline
\end{tabular}

* La correlación es significante al nivel 0,05 (bilateral).

** La correlación es significativa al nivel 0,01 (bilateral).

Tabla 6. Correlación entre el tiempo de hospitalización y espacios para realizar actividades

Espacios

\begin{tabular}{|c|c|c|}
\hline \multirow[t]{3}{*}{ Habitación } & Correlación de Pearson &,- 074 \\
\hline & Sig. (bilateral) &, 475 \\
\hline & $\mathrm{N}$ & 96 \\
\hline \multirow[t]{3}{*}{ Sala compartida } & Correlación de Pearson &, $223(*)$ \\
\hline & Sig. (bilateral) &, 029 \\
\hline & $\mathrm{N}$ & 96 \\
\hline \multirow[t]{3}{*}{ Pasillos } & Correlación de Pearson &, 165 \\
\hline & Sig. (bilateral) & 109 \\
\hline & $\mathrm{N}$ & 96 \\
\hline \multirow[t]{3}{*}{ Otros } & Correlación de Pearson &, 185 \\
\hline & Sig. (bilateral) &, 072 \\
\hline & $\mathrm{N}$ & 96 \\
\hline
\end{tabular}

* La correlación es significante al nivel 0,05 (bilateral).

** La correlación es significativa al nivel 0,01 (bilateral). 
Tabla 7. Correlación entre tiempo de hospitalización y la preferencia de supuestas actividades de animación en el hospital

Preferencias

\begin{tabular}{|c|c|c|}
\hline \multirow[t]{3}{*}{ Acompañamiento por profesionales } & Correlación de Pearson &, $210(*)$ \\
\hline & Sig. (bilateral) &, 040 \\
\hline & $\mathrm{N}$ & 96 \\
\hline \multirow[t]{3}{*}{ Grupos de charla guiada } & Correlación de Pearson &, 043 \\
\hline & Sig. (bilateral) &, 676 \\
\hline & $\mathrm{N}$ & 96 \\
\hline \multirow{3}{*}{ Lectura y comentarios } & Correlación de Pearson &, 167 \\
\hline & Sig. (bilateral) &, 105 \\
\hline & $\mathrm{N}$ & 96 \\
\hline \multirow[t]{3}{*}{ Proyección de películas } & Correlación de Pearson &, 014 \\
\hline & Sig. (bilateral) &, 890 \\
\hline & $\mathrm{N}$ & 96 \\
\hline \multirow[t]{3}{*}{ Conferencias } & Correlación de Pearson &, $307(* *)$ \\
\hline & Sig. (bilateral) &, 002 \\
\hline & $\mathrm{N}$ & 96 \\
\hline \multirow[t]{3}{*}{ Juegos de mesa } & Correlación de Pearson & , 133 \\
\hline & Sig. (bilateral) &, 197 \\
\hline & $\mathrm{N}$ & 96 \\
\hline \multirow[t]{3}{*}{ Risoterapia } & Correlación de Pearson &,- 076 \\
\hline & Sig. (bilateral) &, 464 \\
\hline & $\mathrm{N}$ & 96 \\
\hline \multirow[t]{3}{*}{ Actividades con radio o TV } & Correlación de Pearson &, 178 \\
\hline & Sig. (bilateral) &, 082 \\
\hline & $\mathrm{N}$ & 96 \\
\hline \multirow[t]{3}{*}{ Actividades con ordenador o Internet } & Correlación de Pearson &, 028 \\
\hline & Sig. (bilateral) &, 786 \\
\hline & $\mathrm{N}$ & 96 \\
\hline \multirow[t]{3}{*}{ Celebración fiestas } & Correlación de Pearson &, $363(* *)$ \\
\hline & Sig. (bilateral) &, 000 \\
\hline & $\mathrm{N}$ & 96 \\
\hline \multirow[t]{3}{*}{ Actuaciones famosos } & Correlación de Pearson &, 123 \\
\hline & Sig. (bilateral) &, 234 \\
\hline & $\mathrm{N}$ & 96 \\
\hline
\end{tabular}


Tabla 7. Correlación entre tiempo de hospitalización y la preferencia de supuestas actividades de animación en el hospital (cont.)

Preferencias

Visitas de pacientes adultos

a pacientes pediátricos
Correlación de Pearson

Sig. (bilateral)

$\mathrm{N}$
, 135

,189

Realización de periódicos o revistas

\begin{tabular}{lc} 
Correlación de Pearson &,- 091 \\
\hline Sig. (bilateral) &, 376 \\
\hline $\mathrm{N}$ & 96
\end{tabular}

Talleres (dibujo, barro...)

Correlación de Pearson , 036

Sig. (bilateral) , 731

$\mathrm{N}$

96

* La correlación es significante al nivel 0,05 (bilateral).

** La correlación es significativa al nivel 0,01 (bilateral).

TABla 8. Correlación entre tiempo de hospitalización y posibles consecuencias de la oferta de actividades de animación en el paciente

Consecuencias

Elevar estado de ánimo

Correlación de Pearson

Sig. (bilateral)

, 004

$\mathrm{N}$

,971

Reducir estrés y ansiedad

Correlación de Pearson

96

Compensar pensamientos negativos

Sig. (bilateral)

$-, 066$

$\mathrm{N}$

Correlación de Pearson

Sig. (bilateral)

$\mathrm{N}$

Favorecer comunicación

Correlación de Pearson

Sig. (bilateral)

$\mathrm{N}$

Actividad e independencia

Correlación de Pearson

Sig. (bilateral)

$\mathrm{N}$

Aliviar dedicación de los

Correlación de Pearson

, 523

96

, 198

, 053

96 ,336(**) , 001 96 , 016 , 878

Sig. (bilateral)

$\mathrm{N}$

96 , 165 , 109 96 
Tabla 8. Correlación entre tiempo de hospitalización y posibles consecuencias de la oferta de actividades de animación en el paciente (cont.)

Consecuencias

\begin{tabular}{|c|c|c|}
\hline \multirow[t]{3}{*}{ Mejorar adaptación } & Correlación de Pearson &, $252\left(^{*}\right)$ \\
\hline & Sig. (bilateral) & ,013 \\
\hline & $\mathrm{N}$ & 96 \\
\hline \multirow[t]{3}{*}{ Ofertar atención integral } & Correlación de Pearson &, $225\left(^{*}\right)$ \\
\hline & Sig. (bilateral) &, 027 \\
\hline & $\mathrm{N}$ & 96 \\
\hline \multirow[t]{3}{*}{ Otros } & Correlación de Pearson &, 173 \\
\hline & Sig. (bilateral) & ,092 \\
\hline & $\mathrm{N}$ & 96 \\
\hline
\end{tabular}

* La correlación es significante al nivel 0,05 (bilateral).

** La correlación es significativa al nivel 0,01 (bilateral).

\section{Referencias bibliográficas}

BADOS, A. (2004). La política sociocultural de carácter público: necesidades y alternativas. En: Ministerio de Educación, Cultura y Deporte, Servicios Socioculturales (ed.), La cultura del ocio. Aulas de Verano. Madrid: MEC y D, 31-59.

BAYÉs, R. (1998). Psicología del sufrimiento y de la muerte. Anuario de Psicología, Vol. 29, 4, 5-17. Bermúdez, M. T. (2006). La animación hospitalaria. En: V. Ventosa (coord.), Perspectivas actuales de la Animación sociocultural. Madrid: CCS, 331-343.

Bermúdez, M. T. (2010). La animación hospitalaria con personas mayores. Revista de Ciencias de la Educación, 221, 93-107.

Bermúdez, M. T. y Torío, S. (2007). El voluntariado y la animación hospitalaria. En: X. M. Cid, y A. Peres (ed.), Educación Social, Animación Sociocultural y Desarrollo Comunitario. Universidad de Vigo, Universidad de Trás-os-Montes e Alto Douro y Sociedad Iberoamericana de Pedagogía Social, 589-603.

Costa Ferrer, M. (coord.) (2000). El juego y el juguete en la hospitalización infantil. Valencia: Nau Llibres.

FernÁndez, M. C.; Torío, S. y Bermúdez, M. T. (2010). Animación hospitalaria con pacientes adultos en el Hospital Universitario Central de Asturias: un estudio de necesidades. Proyecto financiado por la Universidad de Oviedo.

Manzanera, M. P.; Ullán, A. M. y Gómez, S. (2008). Animación sociocultural hospitalaria: experiencia de talleres de fotografía y radio en el Hospital Universitario de Salamanca. En: V. VentosA (coord.), Los agentes de la Animación Sociocultural. Madrid: CCS, 641-656.

Peterson, C., Seligman, M. E. y Vaillant, G. (1988). Pessimistic explanatory style as a risk factor for physical illness. A thirty-five year longitudinal study. Journal of Personality and Social Psychology, 55, 23-27.

SARrate, M. L. (1998). Nuevos horizontes de la animación sociocultural. II Congreso Estatal de Educación Social «La Educación Social ante la sociedad en cambio». Madrid.

Ullán, A. M. y Belver, M. H. (2008). Cuando los pacientes son niños. Humanización y calidad en la hospitalización pediátrica. Madrid: Eneida. 
Animación hospitalaria con pacientes adultos en el Hospital la Fe de Valencia: Un estudio de necesidades

Ullán, A. M.; Serrano, I.; Badía, R. y Delgado, J. (2010). Hospitales amigables para adolescentes: preferencias de los pacientes. Enfermería Clínica, 20 (6), 341-348.

Vera, B. (2006). Psicología Positiva: una nueva forma de entender la Psicología. Papeles del Psicólogo, vol. 27 (1), 3-8.

\section{Fuentes electrónicas}

Gonzalo, S.; Gonzalo, J.; Montesinos, S. y de Gracia, M. (2008). Las actividades de ocio en unidades de hospitalización psiquiátrica de agudos como terapia para el fomento de la salud, reducción del tiempo de ingreso y preparación al alta. Enfermería en Psiquiatría y Salud Mental. $9^{\circ}$ Congreso Virtual de Psiquiatría. Interpsiquis-2. http:// www.psiquiatria.com (Fecha de consulta 20/12/2011).

Martínez, S. y Amayra, I. (2006). Beneficios del ocio en la vejez: pautas para el desarrollo de programas de ocio terapéuticos. $7^{\circ}$ Congreso Virtual de Psiquiatría. Interpsiquis. http://www.psiquiatría.com/artículos/psicogeriatría/25043 (Fecha de consulta: 20/12/2011).

\section{Abstract}

Socio-cultural animation with adult patients at Hospital La Fe (Valencia. Spain): a needs analysis

INTRODUCTION. This paper showcases the results of a research study on hospital socio-animation conducted among adult patients at La Fe Hospital (Valencia. Spain) whose aim was to stress the need for these patients to occupy their free time. METHODS. A questionnaire regarding the use of free time by in-patients, a proposal of socio-cultural activities and their consequences was administered to a 96 patient sample. RESULTS. Positive correlations were found between the age of the patients and their lack of interest in taking part in proposed hospital activities and negative correlations were found between their age and their preferences for these activities. Similarly, positive correlations were found between hospitalization periods and the use of their spare time (strolling, chatting), between hospitalization periods and their preference for the cultural offer and professional guidance, and between these periods and the consequences perceived by patients. DISCUSSION. An inverse relationship can be established between the interest of the patients in carrying out activities and the age of the patients.

Key words: Socio-cultural animation, Leisure, Patients, Adults.

\section{Résumé}

Animation en milieu hospitalier chez des patients adultes à l'hôpital de La Fe (Valencia, Espagne): une analyse des besoins

INTRODUCTION. On expose dans cet article les résultats d'une étude réalisée à propos des activités d'animation en milieu hospitalier chez des patients adultes à l'hôpital de La Fe (Valencia, Espagne), qui avait pour but de montrer la nécessité d'occuper leur temps libre. MÉTHODE. Un questionnaire a été appliqué à un échantillon de 96 patients, qui portait sur l'emploi de leur temps libre à l'hôpital, sur l'offre d'activités d'animation et sur les conséquences qui pourraient en résulter. 
RÉSULTATS. On a obtenu des corrélations positives entre l'âge des patients et leur désintérêt pour les activités proposées, et des corrélations négatives entre l'âge et la préférence pour l'offre d'activités. On a obtenu également des corrélations positives entre le temps d'hospitalisation et l'emploi du temps libre (se promener, discuter); de même entre le temps d'hospitalisation et la préférence pour l'offre d'activités de diffusion culturelle et les accompagnements professionnels; et aussi entre ce temps-là et les conséquences perçues par le patient. CONCLUSION. On constate l'intérêt des patients pour la réalisation d'activités en proportion inverse à leur âge.

Mots clés: Animation socioculturelle, Récréation, Patients, Adultes.

\section{Perfil profesional de las autoras}

\section{Ma Teresa Bermúdez Rey}

Profesora de Animación Sociocultural I y II en el Grado de Educación Social (EUPO). Universidad de Oviedo. Doctora en Psicología, ha realizado investigaciones en el campo de la animación hospitalaria con pacientes pediátricos y adultos en el Hospital Universitario Central de Asturias y con adultos en el Hospital de La Fe de Valencia. Ponente invitado sobre el mismo tema en distintos congresos organizados desde el Grado de Animación Sociocultural en la Universidad de Tras Os Montes e Alto Douro (UTAD) en Portugal.

Correo electrónico de contacto: bermudezteresa@uniovi.es, t.bermu@eupo.es

\section{$\mathbf{M}^{\text {a }}$ Eugenia Martín Palacio}

Profesora Ayudante Doctor de Psicología Evolutiva y de la Educación de la Universidad Complutense de Madrid. Doctora en Psicología por la Universidad de Oviedo. Acreditada por la ANECA y ACAP como profesor Ayudante Doctor y Contratado Doctor. Ha publicado en revistas ISI y ha sido ponente en congresos nacionales e internacionales. Ha realizado estancias académicas en la Universidad de Playa Ancha (Chile).

Correo electrónico de contacto: mariaeugeniamartin@edu.ucm.es

\section{Silvia Castellanos Cano}

Licenciada en Psicología, Becaria predoctoral de la Universidad de Oviedo. Investiga en el área de Orientación Educacional (evaluación de competencias personales y sociales y desarrollo de programas en el ámbito del procesamiento estratégico de la información). Ha presentado distintas comunicaciones en congresos iberoamericanos e internacionales de Psicología y publicado en el International journal of developmental and educational psychology.

Correo electrónico de contacto: silviaccano@gmail.com 